\title{
Non-uniform phases in metals with local moments
}

\author{
Angsula Ghosh ${ }^{1,2, *}$ and E. Miranda ${ }^{1,0}$ \\ ${ }^{1}$ Instituto de Física Gleb Wataghin, Unicamp, Caixa Postal 6165, Campinas SP 13083-970, Brazil \\ ${ }^{2}$ Department of Theoretical Physics, Indian Association for the Cultivation of Science, Kolkata 700032, India
}

(Dated: 4th January 2019)

\begin{abstract}
The two-dimensional Kondo lattice model with both nearest and next-nearest neighbor exchange interactions is studied within a mean-field approach and its phase diagram is determined. In particular, we allow for lattice translation symmetry breaking. We observe that the usual uniform inter-site order parameter is never realized, being unstable towards other more complex types of order. When the nearest neighbor exchange $J_{1}$ is ferromagnetic the flux phase is always the most stable state, irrespective of the value of the next-nearest-neighbor interaction $J_{2}$. For antiferromagnetic $J_{1}$, however, either a columnar or a flux phase is realized, depending on conduction electron filling and the value of $J_{2}$.
\end{abstract}

PACS numbers: 75.30.Mb, 71.27.+a, 75.20.Hr

The nature of the various magnetic phases of heavy fermion compounds has been the focus of attention over the years. Most of the analysis is based on the celebrated paradigm of Doniach, who conjectured a phase diagram consisting of two possible phases, one paramagnetic and another exhibiting long range antiferromagnetic order. ${ }^{1}$ The driving mechanism behind this phase diagram is the competition between the Kondo effect, $\frac{2}{\underline{2}}$ which favors paramagnetism and is dominant at strong exchange coupling, and the Ruderman-Kittel-Kasuya-Yosida (RKKY) interaction, ${ }^{3.4 .5}$ which dominates at weak coupling and can lead to antiferromagnetism. Particularly interesting is the quantum phase transition which separates the two phases at zero temperature and which can be accessed by tuning the exchange interaction between local moments and conduction electrons through external or chemical pressure. This quantum critical behavior has been intensively studied experimentally ${ }^{6.7 .8}$ but a complete theoretical description is still lacking ${ }^{9.10 .11 .12 .13 .14}$

Despite the appealing simplicity of the Doniach phase diagram the possibility of the existence of other kinds of phases remains. Among these we should mention inhomogeneous magnetic order ${ }^{15}$ orbital antiferromagnetism ${ }^{16}$ and dimerization. ${ }^{17}$ The last possibility has been given strong numerical support in the one-dimensional case at quarter conduction electron filling. 17 It was ultimately ascribed to the long-ranged RKKY interaction between localized spins $\frac{17}{17}$ Although dimerization is an oft-encountered instability in one dimension, its presence in higher dimensions is less frequent. There is some (controversial) evidence in favor of its existence in the frustrated two-dimensional Heisenberg model with both nearest- and next-nearest-neighbor interactions. $\frac{18.19,20.21}{}$ However, the long-ranged nature of the RKKY interaction makes its appearance more likely in metallic systems with local moments. Motivated by this, the aim of the present study is to look for dimerization in particular and other forms of order with broken lattice translational symmetry in general in higher dimensional models of heavy fermion materials.

The co-existence of magnetic inter-site correlations and the Kondo effect has been investigated before using mean field calculations. Usually, two order parameters are considered: one describing the local correlations generated by the Kondo effect and the other connected to non-local inter-site correlations. If the inter-site correlations break spin $\mathrm{SU}(2)$ symmetry, there is a competition between Kondo singlet formation and magnetic ordering of some type. ${ }^{22,23,24}$ Alternatively, the tendency for Kondo compensation can be analyzed in a scaling approach. ${ }^{25,26.27}$ On the other hand, if the inter-site correlations do not break spin $\mathrm{SU}(2)$ symmetry, there may be the formation of some kind of spin liquid state. ${ }^{28.29 .30}$ Fluctuations beyond mean field have also been considered in connection with the quantum critical behavior of the system ${ }^{31.32}$ In this study, we have allowed for the emergence of broken lattice translational symmetry in the non-local correlations, without a broken $\mathrm{SU}(2)$ symmetry. We have studied the effects of conduction electron filling and both nearest- and next-nearest-neighbor exchange interactions on the possible phases of the Kondo lattice model in two dimensions. The inclusion of further-neighbor interactions is intended to partially incorporate the long-ranged nature of the RKKY interaction between localized moments. We have found that the usually assumed uniform state is unstable throughout the phase diagram towards either columnar or flux phase order ${ }^{33.34}$ We have also studied the temperature dependence of the order parameters. They do not seem to differ much from the uniform case $^{30}$

The Kondo lattice Hamiltonian is given by

$$
H_{K}=\sum_{\mathbf{k} \sigma}\left(\epsilon_{\mathbf{k}}-\mu\right) c_{\mathbf{k} \sigma}^{\dagger} c_{\mathbf{k} \sigma}+J_{K} \sum_{j, \alpha \beta} \mathbf{S}_{j} \cdot c_{j \alpha}^{\dagger} \boldsymbol{\sigma}_{\alpha \beta} c_{j \beta},
$$

where $\epsilon_{\mathbf{k}}$ is the band dispersion, $\mu$ is the chemical potential, $c_{j \sigma}$ and $c_{\mathbf{k} \sigma}$ are conduction electron annihilation operators in real (Wannier) and reciprocal spaces respectively, $\mathbf{S}_{j}$ is a localized spin- $\frac{1}{2}$ operator, and $\boldsymbol{\sigma}_{\alpha \beta}$ are Pauli matrices. In addition to the above terms we also include Heisenberg-like interactions between nearest neighbor and next-nearest neighbor localized spins in an 
attempt to partially capture the long-ranged nature of the RKKY interaction. Hence, the full Hamiltonian can now be written as $H=H_{K}+H_{H}$, where

$$
H_{H}=J_{1} \sum_{\langle j k\rangle} \mathbf{S}_{j} \cdot \mathbf{S}_{k}+J_{2} \sum_{\langle\langle l m\rangle\rangle} \mathbf{S}_{l} \cdot \mathbf{S}_{m},
$$

where $\langle j k\rangle$ and $\langle\langle l m\rangle\rangle$ denote nearest-neighbor and nextnearest neighbor sites, respectively. In this work, we consider both ferromagnetic and antiferromagnetic exchange interactions. The spin operators can be expressed in the usual Abrikosov pseudo-fermionic representation

$$
\mathbf{S}_{j}=\frac{1}{2} f_{j \alpha}^{\dagger} \boldsymbol{\sigma}_{\alpha \beta} f_{j \beta},
$$

where a constraint of single $f$-electron occupancy is implied. The mean-field Hamiltonian can be written by expressing the spin fields in terms of the above $f$-fermionic operators and defining the following three order parameters

$$
\begin{aligned}
\phi_{j \sigma} & \equiv \frac{1}{2}\left\langle c_{j \sigma}^{\dagger} f_{j \sigma}+f_{j \sigma}^{\dagger} c_{j \sigma}\right\rangle, \\
\chi_{j k \sigma} & \equiv \frac{1}{2}\left\langle f_{j \sigma}^{\dagger} f_{k \sigma}+f_{k \sigma}^{\dagger} f_{j \sigma}\right\rangle, \\
\chi_{l m \sigma}^{\prime} & \equiv \frac{1}{2}\left\langle f_{l \sigma}^{\dagger} f_{m \sigma}+f_{m \sigma}^{\dagger} f_{l \sigma}\right\rangle,
\end{aligned}
$$

where $j$ and $k$ are nearest neighbor sites and $l$ and $m$ denote next-nearest neighbors. We will focus on $\mathrm{SU}(2)$ invariant states, hence none of the order parameters will depend on $\sigma\left(\phi_{j \sigma}=\phi_{j}, \chi_{j k \sigma}=\chi_{j k}, \chi_{l m \sigma}^{\prime}=\chi_{l m}^{\prime}\right)$. We can write down the mean field Hamiltonian as

$$
\begin{aligned}
H_{M F} & =\sum_{\mathbf{k}, \sigma}\left(\epsilon_{\mathbf{k}}-\mu\right) c_{\mathbf{k} \sigma}^{\dagger} c_{\mathbf{k} \sigma}+E_{0} \sum_{j \sigma} f_{j \sigma}^{\dagger} f_{j \sigma} \\
& -2 J_{K} \sum_{j, \sigma} \phi_{j}\left(c_{j, \sigma}^{\dagger} f_{j, \sigma}+\text { H.c. }\right) \\
& -J_{1} \sum_{\langle j k\rangle, \sigma}\left(\chi_{j k} f_{j, \sigma}^{\dagger} f_{k, \sigma}+\text { H. c. }\right) \\
& -J_{2} \sum_{\langle\langle l m\rangle\rangle, \sigma}\left(\chi_{l m}^{\prime} f_{l, \sigma}^{\dagger} f_{m, \sigma}+\text { H. c. }\right) \\
& +4 J_{K} \sum_{j}\left|\phi_{j}\right|^{2}+2 J_{1} \sum_{\langle j k\rangle}\left|\chi_{j k}\right|^{2}+2 J_{2} \sum_{\langle\langle l m\rangle\rangle} \mid \chi_{l m}^{\prime}\langle\dot{6})
\end{aligned}
$$

We will focus on a two-dimensional tight-binding dispersion relation for the conduction band

$$
\epsilon_{\mathbf{k}}=-\frac{D}{2}\left(\cos k_{x} a+\cos k_{y} a\right),
$$

where $D$ is the half bandwidth and $a$ is the lattice parameter. The chemical potential is determined by the conduction electron density $n$ through $\frac{1}{N} \sum_{\mathbf{k} \sigma}\left\langle c_{\mathbf{k} \sigma}^{\dagger} c_{\mathbf{k} \sigma}\right\rangle=n$ ( $N$ is the number of lattice sites) and $E_{0}$ is a Lagrange multiplier used to impose the $f$-electron single occupancy (a)

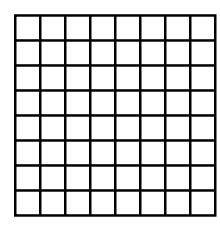

(c)

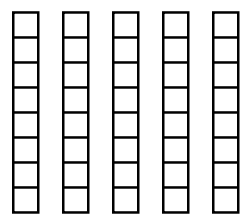

(b)

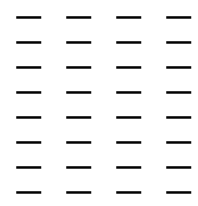

(d)

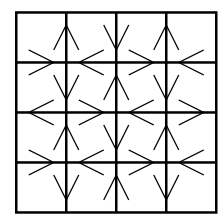

Figure 1: Schematic picture illustrating the various possible phases: (a) Uniform phase, (b) Dimer phase, (c) Columnar phase, and (d) Flux phase.

constraint on the average $\frac{1}{N} \sum_{\mathbf{k} \sigma}\left\langle f_{\mathbf{k} \sigma}^{\dagger} f_{\mathbf{k} \sigma}\right\rangle=1$. The free energy can be written as

$$
\begin{aligned}
F & =-2 T \sum_{\mathbf{k}, \alpha= \pm} \ln \left[1+e^{-E_{\mathbf{k}}^{\alpha} / T}\right]+\left(E_{0}-\mu n\right) N \\
& +4 J_{K} \sum_{j}\left|\phi_{j}\right|^{2}+2 J_{1} \sum_{\langle j k\rangle}\left|\chi_{j k}\right|^{2}+2 J_{2} \sum_{\langle\langle l m\rangle\rangle}\left|\chi_{l m}^{\prime}\right|^{2}(8)
\end{aligned}
$$

where $T$ is the temperature and $E_{\mathbf{k}}^{ \pm}$are the noninteracting bands of the mean field Hamiltonian (6).

In this work, we have chosen energy and length units such that both $D$ and $a$ are equal to 1 . When translational invariance is not broken, $\phi_{j}=\phi, \chi_{j k}=\chi$ and $\chi_{l m}^{\prime}=\chi^{\prime}$, which we will henceforth call the uniform state. In addition to the uniform case, we also consider the dimerized state with dimers along the x-axis, the columnar phase and the flux phase ${ }^{33.34}$ In all cases, $\phi_{j}$ and $\chi_{l m}^{\prime}$ are taken to be uniform. The four phases are described as follows (see Fig. 11):

(a) Uniform: All $\chi$ 's are real and equal. Lattice translation symmetry is not broken.

(b) Dimers: $\chi$ is zero for bonds along the $y$-direction whereas for bonds along the $x$-direction we have

$$
\chi_{j k}=\frac{\chi}{2}\left[1+(-1)^{j}\right] .
$$

This phase has broken lattice translational and rotational symmetries. Another state with the $x$ and $y$ directions interchanged is degenerate and equivalent to this one.

(c) Columnar: $\chi$ is uniform and equal to $\chi$ for bonds along the $y$-direction whereas for bonds along the $x$ direction we have

$$
\chi_{j k}=\frac{\chi}{2}\left[1+(-1)^{j}\right] .
$$

This phase also has broken lattice translational and rotational symmetries. By interchanging $x$ and $y$ directions we once again get another degenerate and equivalent state.

(d) Flux Phase: All of $\chi$ 's are equal in magnitude 


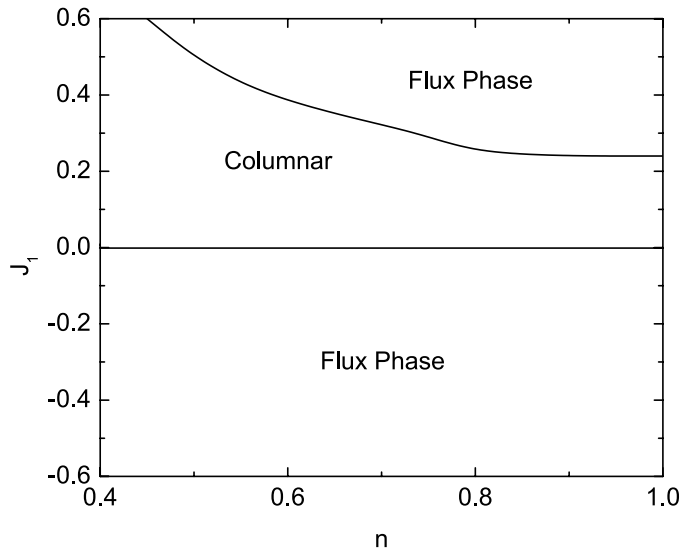

Figure 2: Phase diagram of the Kondo-Heisenberg model at $T=0$ as a function of the nearest-neighbor exchange $J_{1}$ and the conduction electron filling $n$. The next-nearest-neighbor coupling $J_{2}=0$ and the Kondo coupling $J_{K}=0.5$.

but may have imaginary phases. The specific choice of these phases is not gauge-invariant 34 However, the flux through a plaquette is a gauge-invariant quantity. It is given by the phase of the oriented plaquette product $\Pi=\chi_{12} \chi_{23} \chi_{34} \chi_{41},{ }^{34}$ We consider the case depicted in Fig. $1(\mathrm{~d})$, in which $\prod= \pm$, staggered between adjacent plaquettes, corresponding to fluxes of $\pm \pi$. This choice can be realized by the following gauge choice

$$
\chi_{j k}=|\chi|
$$

for bonds along the $y$-axis and

$$
\chi_{j k}=(-1)^{j} i|\chi|
$$

for bonds along the $x$-axis. The bonds now being complex have a definite direction which is shown in Fig. 11(d).

We first study the phase diagram of the model at $T=0$ by varying $J_{1}$ and $n$ while keeping $J_{2}=0$. The Kondo coupling is kept at $J_{K}=0.5$. This is shown in Fig 2 We consider both ferromagnetic and antiferromagnetic values of $J_{1}$. The first thing to notice is the instability of the uniform state, which is usually assumed, towards other forms of order. For antiferromagnetic coupling between the local moments the columnar and flux phases are the most stable, the latter occurring only for sufficiently large $J_{1}$. However, when $J_{1}<0$ (ferromagnetic coupling), the flux phase is the most stable, irrespective of the filling and the value of $J_{1}$. The transition between flux and columnar phase is first order.

We now proceed to investigate the influence of the next-nearest-neighbor coupling $J_{2}$ between the local moments, still at $T=0$. We studied the phase diagram at $n=0.9$ and $n=0.4$ (Figs. 3 and 4] respectively). We have allowed for both antiferromagnetic and ferromagnetic couplings between next-nearest neighbors. For ferromagnetic $J_{1}$, the flux phase is dominant irrespective of

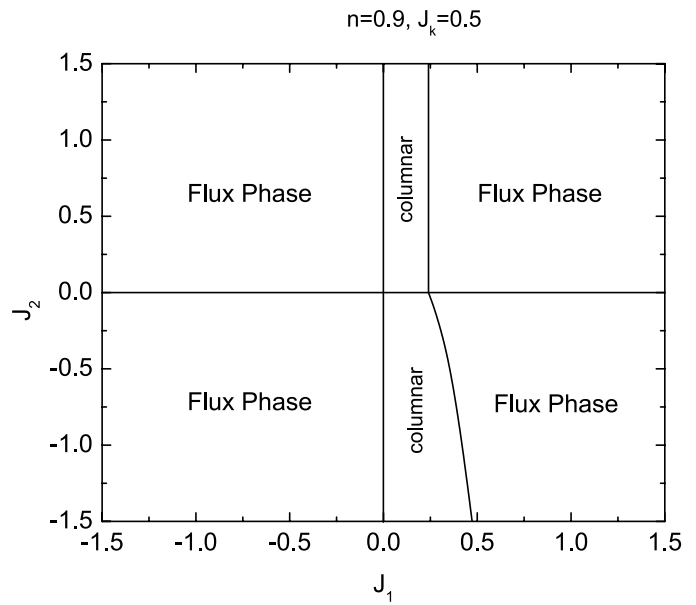

Figure 3: Phase diagram of the Kondo-Heisenberg model at $T=0$ with nearest-neighbor and next-nearest neighbor exchange at $n=0.9$ and $J_{K}=0.5$.

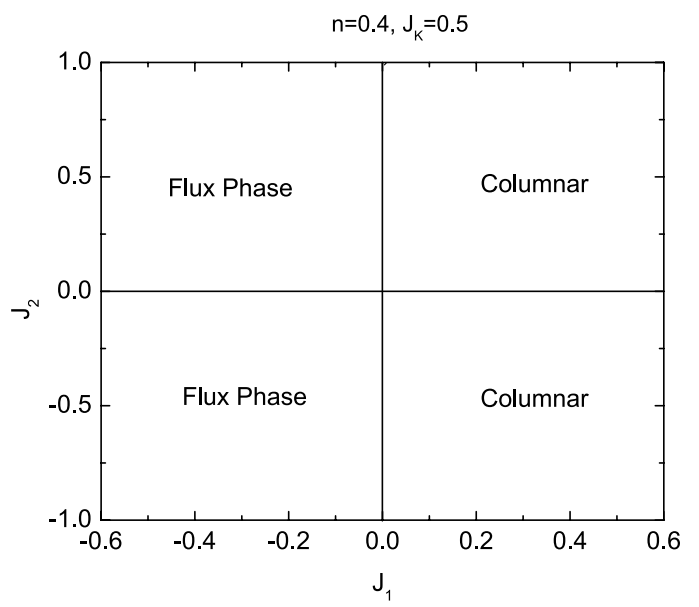

Figure 4: Phase diagram of the Kondo-Heisenberg model at $T=0$ with nearest-neighbor and next-nearest neighbor exchange at $n=0.4$ and $J_{K}=0.5$.

the value of $J_{2}$. For antiferromagnetic $J_{1}$, columnar and flux phases share the parameter space. For $J_{1} \leq 0.24$, only the columnar phase is realized. For higher values of $J_{1}$, a flux phase can appear if the conduction electron filling is large enough, as shown in Fig. 3. At $n=0.4$, on the other hand, the most stable ground state is determined solely by the sign of $J_{1}$, irrespective of the value of $J_{2}$. In this case, a ferromagnetic $J_{1}$ favors the flux phase, whereas an antiferromagnetic $J_{1}$ leads to a columnar phase. Again, the phase boundary between flux and columnar phases is a first order line.

In Fig. 5 we show the filling dependence of the order parameters $\phi$ and $\chi$ at $T=0$, for $J_{1}=0.2, J_{2}=0$, and $J_{K}=0.5$. In this case, the system is always in a columnar 


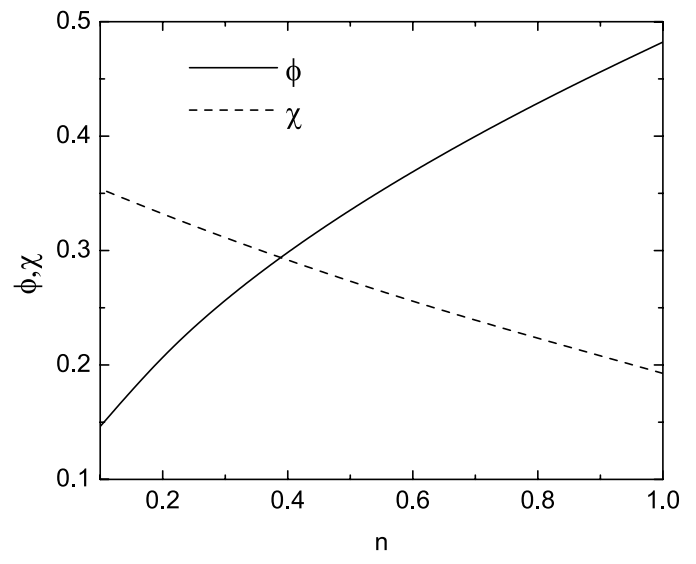

Figure 5: Filling dependence of the order parameters $\phi$ and $\chi$ at $T=0$ in the Kondo-Heisenberg model with nearestneighbor exchange only $\left(J_{1}=0.2\right)$ and $J_{K}=0.5$.

phase (see Fig. 21). There is a clear competition between the two types of order, the Kondo effect $(\phi)$ becoming more predominant as the system approaches half-filling. Of course, this competition is analogous to the one predicted by Doniach between a tendency to form to local singlets $(\phi)$ and another one to lock localized spins into some kind of order. Our mean field Ansatz is able to capture this competition. The predominance of the Kondo effect as the system approaches half-filling is due to an enhanced density of states in that region providing more conduction electron states to quench the local moments. By contrast, note that, for the same parameters of Fig. [5 the uniform order parameter has a much more reduced value and does not compete with the Kondo effect at the mean field level (see Fig. 3 of Ref ${ }^{30}$ ).

In addition, we have also studied the temperature dependence of the order parameters for $J_{1}=0.2, J_{2}=0$, and $J_{K}=0.5$. The temperature dependence has been plotted for $n=0.8$ in Fig. [6] and $n=0.4$ in Fig. [7] In both cases, the columnar phase is the most stable from $T=0$ up to the transition temperature. Although the two dependences are different, both order parameters $\phi$ and $\chi$ disappear at the same critical temperature. This same simultaneous disappearance of order had been observed in previous studies of the uniform phase for similar values of the exchange couplings ${ }^{30}$ Although the finite temperature phase transition triggered by $\chi$ could be realized in real systems, the vanishing of $\phi$ is an artifact of the mean field treatment 35

Let us now pause to compare our results with previous studies. A mean field Ansatz of the form considered here has been investigated before, $\stackrel{28.29 .30}{ }$ without allowance for broken lattice translation symmetry. An important conclusion of our results is that the uniform state considered in these references is never stable. Refs. 25,2627, on the other hand, do consider the effects of both nearest and next-nearest neighbor couplings between local-

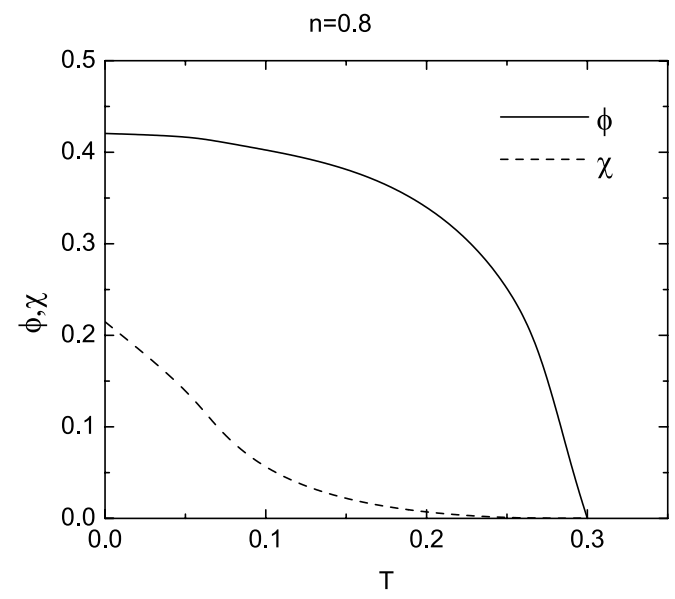

Figure 6: Temperature dependence of the order parameters $\phi$ and $\chi$ (columnar phase) at $n=0.8$ in the Kondo-Heisenberg model with nearest-neighbor exchange only $\left(J_{1}=0.2\right)$ and $J_{K}=0.5$.

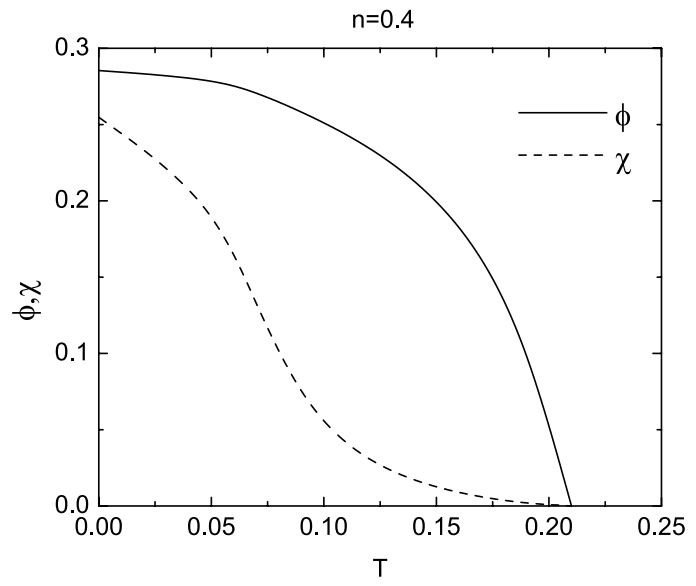

Figure 7: Temperature dependence of the order parameters $\phi$ and $\chi$ (columnar phase) at $n=0.4$ in the Kondo-Heisenberg model with nearest-neighbor exchange only $\left(J_{1}=0.2\right)$ and $J_{K}=0.5$.

ized spins. Their treatment of the Kondo effect, however, is confined to a scaling analysis, which breaks down below the Kondo scale. Our self-consistent treatment of the Kondo effect, by contrast, is able to reach deep into the Kondo singlet formation regime and thus offers a better treatment below the Kondo scale. Finally, no comparison has been attempted with the mean field free energies of phases with conventional long-range magnetic order ${ }^{22.23 .24}$ This would determine the region of stability of these non-uniform phases. We leave this for future studies.

In conclusion we have studied the mean field phase diagram of the two-dimensional Kondo-Heisenberg model with both nearest- and next-nearest-neighbor exchange 
interactions for various values of doping, temperature and coupling constants. We have observed that the uniform state solution is unstable towards lattice translational symmetry breaking for any value of the exchange constants. Depending on the values of $J_{1}, J_{2}$ and filling $n$, the system realizes either a columnar or a flux phase. The flux phase is always stabilized by a nearest-neighbor ferromagnetic exchange between localized spins. When this coupling constant changes sign, however, both columnar and flux phases can occur, the latter being favored at large $J_{1}$ and $n$ and the former appearing at small $J_{1}$ and low fillings.

One of us (A. G.) would like to thank Dr. A. P. Vieira for helpful discussions. The authors would like to thank the financial support of the Brazilian Agencies FAPESP, through grants 01/00719-8 (E. M.) and 02/03799-5 (A. G.), and CNPq, though grant 301222/97-5 (E. M.) and the Indian Agency CSIR (A. G.).
* Electronic address: tpag2@mahendra.iacs.res.in

† Electronic address: emiranda@ifi.unicamp.br

1 S. Doniach, Physica B 91, 231 (1977).

2 A. C. Hewson, The Kondo Problem to Heavy Fermions (Cambrige University Press, Cambridge, 1993).

3 M. A. Ruderman and C. Kittel, Phys. Rev. 96, 99 (1954).

4 T. Kasuya, Progr. Theoret. Phys. 16, 45 (1956).

${ }^{5}$ K. Yosida, Phys. Rev. 106, 893 (1957).

${ }^{6}$ G. R. Stewart, Rev. Mod. Phys. 73, 797 (2001).

${ }^{7}$ H. v. Löhneysen, J. Phys.: Condens. Matter 8, 9689 (1996).

8 A. Schröder, G. Aeppli, R. Coldea, M. Adams, O. Stockert, H.v. Löhneysen, E. Bucher, R. Ramazashvili, and P. Coleman, Nature 407, 351 (2000).

9 T. Moryia, Spin Fluctuations in Itinerant Electron Magnetism (Springer-Verlag, Berlin, 1985).

10 J. A. Hertz, Phys. Rev. B 14, 1165 (1976).

11 A. J. Millis, Phys. Rev. B 48, 7183 (1993).

12 M. A. Continentino, Phys. Rep. 239, 179 (1994).

13 P. Coleman, C. Pépin, Q. Si, and R. Ramazashvili, J. Phys.: Condens. Matter 13, R723 (2001).

14 Q. Si, S. Rabello, K. Ingersent, and J. L. Smith, Nature 413, 804 (2001).

15 K. Matsuda, Y. Kohori, T. Kohara, K. Kuwahara, and H. Amitsuka, Phys. Rev. Lett. 87, 087203 (2001).

16 P. Chandra, P. Coleman, J. A. Mydosh, and V. Tripathi, Nature 417, 831 (2002).

17 J. C. Xavier, R. G. Pereira, E. Miranda, and I. Affleck, Phys. Rev. Lett. 90, 247204 (2003).

18 N. Read and S. Sachdev, Phys. Rev. Lett. 66, 1773 (1991).

19 V. N. Kotov, J. Oitmaa, O. Sushkov, and Z. Weihong, Philos. Mag. B 80, 1483 (2000).
20 L. Capriotti and S. Sorella, Phys. Rev. Lett. 84, 3173 (2000).

21 L. Capriotti, F. Becca, A. Parola, and S. Sorella, Phys. Rev. B 67, 212402 (2003).

${ }^{22}$ V. Yu. Irkhin and M. I. Katsnelson, J. Phys.: Condens. Matter 2, 8715 (1990).

${ }^{23}$ V. Yu. Irkhin and M. I. Katsnelson, Z. Phys. B 82, 77 (1991)

24 M. D. Kim, C. K. Kim, and J. Hong, Phys. Rev. B 68, 174424 (2003).

25 V. Yu. Irkhin and M. I. Katsnelson, Phys. Rev. B 56, 8109 (1997).

26 V. Yu. Irkhin and M. I. Katsnelson, Phys. Rev. B 59, 9348 (1999).

27 V. Yu. Irkhin and M. I. Katsnelson, Phys. Rev. B 61, 14640 (2000).

28 P. Coleman and N. Andrei, J. Phys.: Condens. Matter 1, 4057 (1987).

29 J. R. Iglesias, C. Lacroix, and B. Coqblin, Phys. Rev. B 56, 11820 (1997).

30 A. R. Ruppenthal, J. R. Iglesias, and M. A. Gusmão, Phys. Rev. B 60, 7321 (1999).

31 T. Senthil, S. Sachdev, and M. Vojta, Phys. Rev. Lett. 90, 216403 (2003).

32 T. Senthil, M. Vojta, and S. Sachdev, Phys. Rev. B 69, 035111 (2004).

33 I. Affleck and J. B. Marston, Phys. Rev. B 37, 3774 (1988).

34 J. B. Marston and I. Affleck, Phys. Rev. B. 39, 11538 (1989).

35 P. Coleman, Phys. Rev. B 35, 5072 (1987). 